\title{
Combined Experimental and Computational Investigation of Microstructural Plasticity
}

\author{
L.N. Brewer, W.A. Counts, T.E. Buchheit, C.C. Battaile, J.R. Michael \\ Sandia National Laboratories, Materials Characterization Dept., P.O. Box 5800-MS 1411 \\ Albuquerque, NM 87185-1411
}

A great deal is known about the evolution of plastic deformation fields at the continuum level; however, there is a lack of combined theoretical and experimental investigation of plasticity at the microstructural length scale. Electron backscattered diffraction (EBSD) has been used in numerous studies to investigate the amount and distribution of plastic deformation in metallic microstructures, in particular through the measurement of intragranular misorientation. [1]

This study examines the evolution of plastic deformation in a given microstructural field of view as a function of macroscale strain. Well-annealed tensile bars of high purity Ni were strained in steps of $0,1 \%, 5 \%$, and $10 \%$ strain. At each strain increment, EBSD orientation maps were collected from three fields of view. The distribution of plastic deformation was mapped using an intragrain misorientation mapping method. [2] These misorientation maps were compared to misorientation maps calculated using a local crystal plasticity-based finite element method. [3] The calculations used the initial 0\% strain EBSD orientation map as the starting mesh. This mesh was deformed to $10 \%$ total strain.

The EBSD misorientation maps display several interesting features concerning the microstructural evolution of plasticity. At 1\% strain, the misorientation fields are just observable, particularly along grain boundaries. (Fig. 1A and 2A). At 5\% strain, the deformation fields are readily visible with a somewhat continuous band of elevated misorientation around the perimeter of each grain. (Fig. 1B and 2B) At 10\% strain, some grains maintain the same misorientation distribution around the periphery of the grains, but the misorientation level intensifies (Fig. 1C and $2 \mathrm{C}$ ). In other grains, the misorientation field spreads across the grain. Shear banding is also visible in a couple of the largest grains.

This initial comparison between EBSD measurements and crystal plasticity-based models shows some qualitative agreement but also some important differences in the misorientation distributions (Figure 3). As in the EBSD data, the FEM-derived misorientation maps show the initiation of deformation fields at grain boundaries, but particularly at triple junctions. The FEM results also display the growth of misorientation bands around the outside of grains. However, the absolute level of misorientation in the FEM and EBSD maps differs by at least a factor of two. Additionally, the exact distribution of misorientation along grain boundaries is not in total agreement. This paper will discuss the possible sources for disagreement between the experimental and theoretical results $(2 \mathrm{D}$ versus $3 \mathrm{D}$, modeling of grain boundary resistance to dislocation motion, etc.) and initial work at addressing these areas of disagreement.

1. Kamaya, M., et al. Nuclear Engineering and Design, 2005. 235: p. 713-725.

2. $\quad$ Brewer, L.N., et al. Microscopy and Microanalysis, 2006. 12(85-91): p. 85-91.

3. Buchheit, T.E., et al. International Journal of Plasticity, 2005. 21: p. 221-249.

4. Sandia is a multiprogram laboratory operated by Sandia Corporation, a Lockheed Martin Company, for the United Stated Department of Energy (DOE) under contract DEAC0494AL85000. 
Figure 1. EBSD orientation maps from one area of tensile sample.

A.) $1 \%$ strain

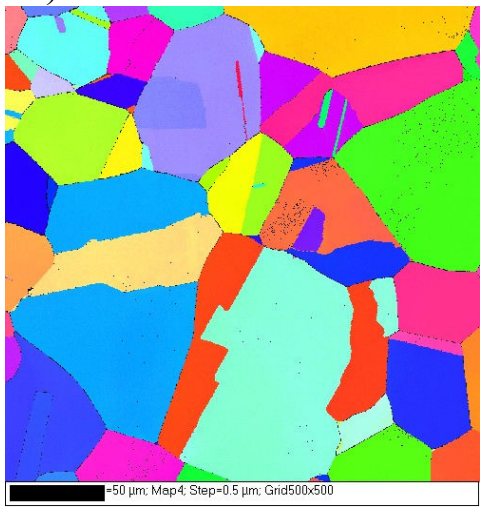

B.) $5 \%$ strain,

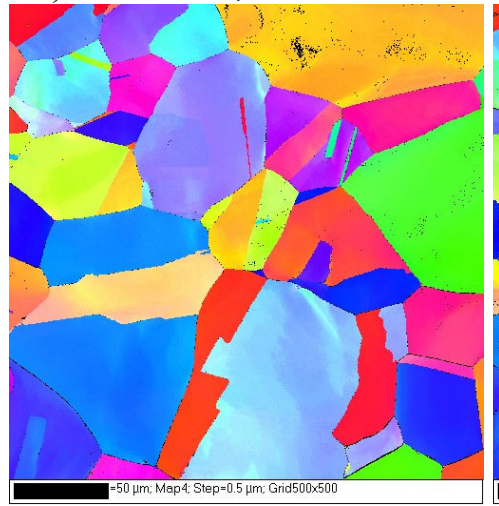

C.) $10 \%$ strain,

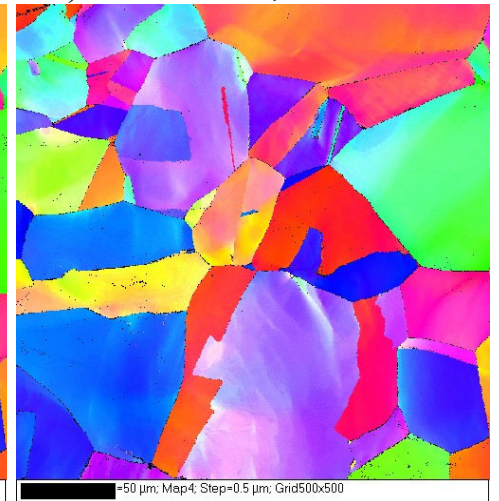

Figure 2. EBSD misorientation maps from one area of sample. Misorientation is given in degrees.

A.) $1 \%$ strain

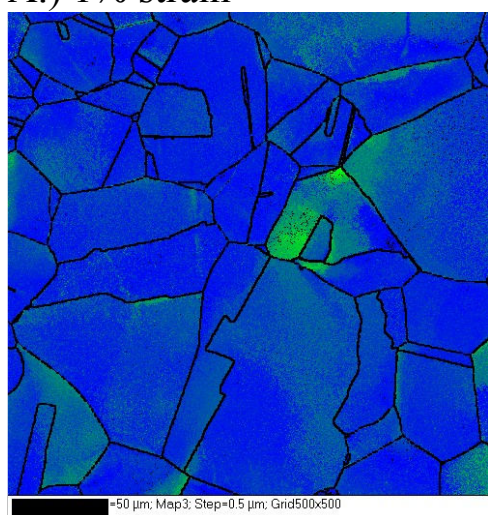

12
B.) $5 \%$ strain,

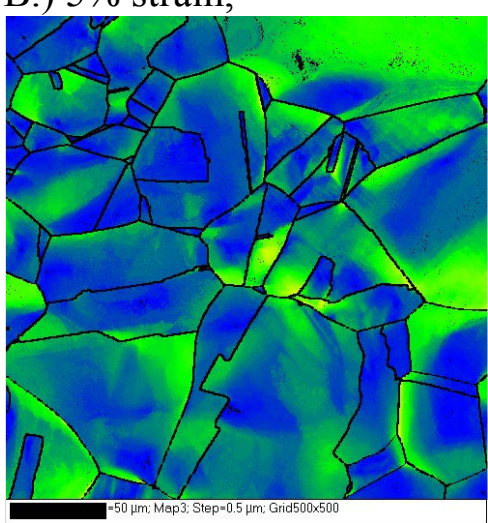

C.) $10 \%$ strain,

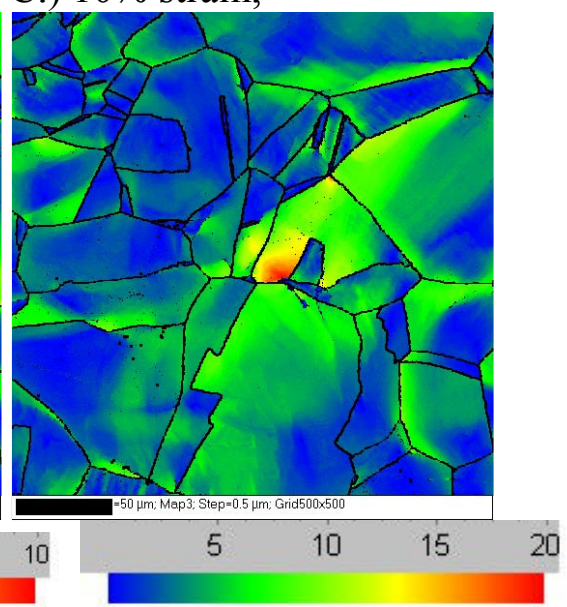

Figure 3. Comparison of local plasticity FEM model with EBSD misorientation maps from the same area. Misorientation angle is given in degrees.

A.) FEM 1\% strain,

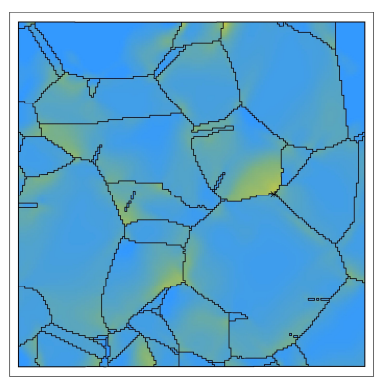

B.) EBSD $1 \%$ strain,

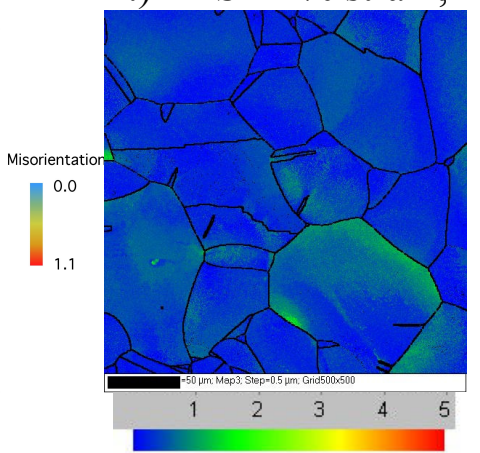

C.) FEM 5\% strain,

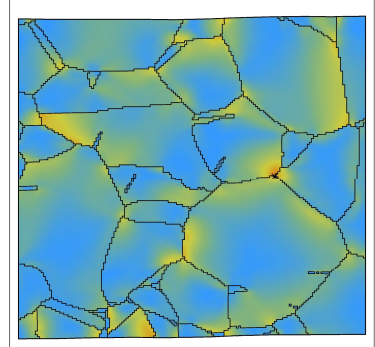

D.) EBSD 5\% strain

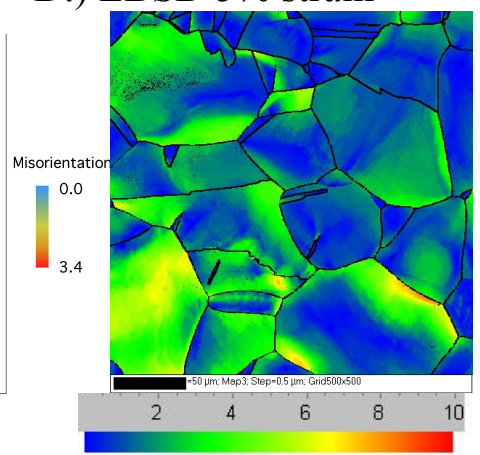

